\title{
Carbon-Containing Waste of Coal Enterprises in Magnetic Sorbents Technology
}

\author{
Ekaterina Kvashevaya $^{1}$, Elena Ushakova $^{1}$, and Andrey Ushakov ${ }^{1 *}$ \\ ${ }^{1}$ T.F. Gorbachev Kuzbass State Technical University, 650000, 28 Vesennyaya St., Kemerovo, Russia
}

\begin{abstract}
The article shows the issues state of coal-mining enterprises carbonaceous wastes utilization, including by obtaining oil-sorbent. The characteristics of the feedstock are presented; experiment methods of obtaining a binder based on the livestock enterprises waste, of forming binder with filler (sawdust, coal waste); of pyrogenetic processing to obtain a sorbent are described. Possible options for the introduction of magnetite (a magnetic component) in the composition of the oil sorbent are considered: on the surface, in the volume of the granule and the magnetite core. In the course of the work it was found that the optimum content of coal dust in the sorbent granules is $75 \%$ by weight, and the most effective way of obtaining the magnetic sorbent is to apply the carbon material directly to the "core" of magnetite. However, in this case, the problem of finding an effective binder for magnetite arises. The option of applying magnetite on the surface of a carbon sorbent is not effective. Thus, at present, we use a mixture of coal waste, which binds to the uniform distribution of magnetite in the volume. The developed magnetic sorbents can be used in various weather conditions, including strong winds and icing of water bodies, as well as for small and medium currents.
\end{abstract}

\section{Introduction}

To date, one of the most large-scale sources of environmental pollution is mining, including coal $-92 \%$ of the total production of waste production according to 2015 [1]. Methods of utilization of coal waste are diverse; the choice of one or another method is based on the composition of the mass, the particle size distribution, and the volume of education.

There are technologies for the use of coal waste in construction, when obtaining rareearth elements and granulated fuel, as raw materials for gasification, etc. [2, 3]. One of the promising directions may be the production of sorbents for cleaning water from petroleum products together with organic binders [4].

According to GREENPEACE in recent years, oil spills are the order of several million tons per year. In this case, there are no clear signs of a reduction in this volume [5]. The International Tanker Owners Pollution Federation (ITOPF) reports a decrease of the amount of oil that has entered water bodies, but it should be noted that spills with a volume of more than 700 tons are included in the statistics $[6,7]$.

\footnotetext{
* Corresponding author: brels@list.ru
} 
Oil is a complex of substances consisting of more than 3000 elements, most of which are easily oxidized, so oil has a rather large toxic effect on plants and living organisms. Another important circumstance is the ability of oil, and especially its light fractions, to spread at high velocity along the water surface, thus forming a thin film of a large area $[8,9]$. Because of this oil spills on the water are considered more dangerous than on the soil, where it is kept for some time by soil particles.

The severity of the consequences of accidents in the water areas is associated with the high rate of oil spread along the water surface, as well as the complexity of the technical means necessary to eliminate the consequences. [10] To reduce the consequences of accidents of spilled oil products, it is necessary to conduct an oil spill operation promptly and with maximum efficiency.

Mechanical methods of removing oil and oil products from the water with sorbents is the most perspective. However, the sorbents currently used do not always meet the requirements for effective liquidation of oil spills. In particular, powder sorbents are difficult to apply to spills, especially in windy weather.

The aim of the work is to develop a composition for obtaining magnetic oil sorbents by medium-temperature pyrolysis based on carbonaceous wastes from industrial enterprises, in particular coal mining waste and coal processing.

\section{Materials and methods}

Experimental studies consisted of the following stages:

1. Analysis of raw components.

2. Binder obtaining based on agricultural waste.

3. Preparation of molded granules from binder, filler and magnetite component.

4. Pyrolysis of the molded granules to obtain a sorbent.

5. Analysis and testing of the finished oil-sorbent.

At the first stage, the properties of the raw materials were studied in accordance with GOST. Coal dust, waste livestock farm, magnetite are used as a raw material

The binder was obtained by anaerobic digestion of the biomass of livestock enterprises in laboratory plants - methane tanks by the methods described in [11].

The experiments on mixtures pelletizing were carried out on a drum-type laboratory granulator, which consisted of a polyethylene drum $0.27 \mathrm{~m}$ in diameter, $0.31 \mathrm{~m}$ in length, and an electromechanical part. The key parameters for mixture pelletizing in a drum were determined experimentally, but at the same time, the roll-over regime was to be maintained, and the yield of the target product fraction was more than $50 \%$ by weight.

The speed of drum rotation ranged from 40 to 140 turnover/min. The required size of the molded granules was achieved by varying the speed of drum rotation: to obtain granules with an average diameter of $5 \mathrm{~mm}-80$ turnover/min, $10 \mathrm{~mm}-60$ turnover/min, $15 \mathrm{~mm}-40$ turnover/min.

The process of granulation took place within 30 minutes. After completion of the granulation, the raw molded granules were dried to constant moisture in a granulator using fans and sent to pyrogenetic processing.

In addition to the "classic" filler - coal fines, the mixture was added magnetite in various ways. Perhaps three models of introducing magnetic particles into the sorbent (Figure 1): "on the surface", "in the bulk" and "magnetite core". 


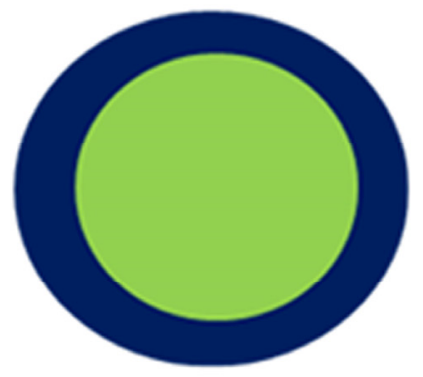

$a$

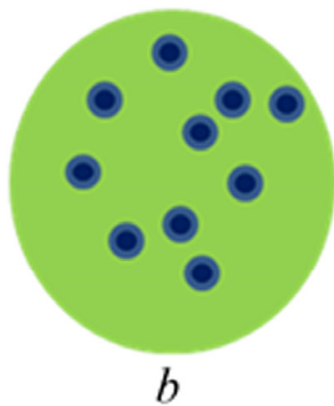

$b$

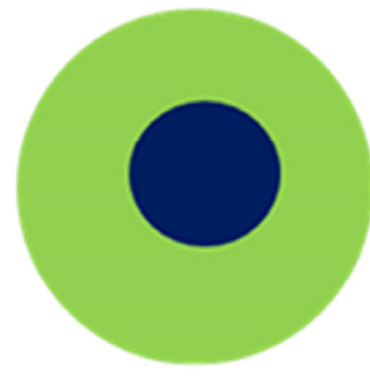

$c$

Fig. 1. Models of introducing magnetic particles into the sorbent: - mixture of filler and binder; - magnetic particles.

The first model provides the location of magnetite on the surface of the molded granules (Figure 1a). With this method, magnetite is not added to the pelletizing mixture, but is introduced in the last stage of granulation by dusting.

The second model (Figure 1b) is the distribution of magnetite particles in the volume of the sorbent. This model is realized at the mixing stage of the binder and filler, where an additional amount of powdered magnetite is additionally introduced.

In the third model (Figure 1c), a mixture of binder and filler is applied to the already formed core of magnetite by the rolling method, that is, the magnetite particles are the centers of granule formation.

The pyrogenetic processing of molded granules consisted in successive pyrolysis and cooling in air or inert gas. The experiments were carried out on an experimental pyrolysis plant (Figure 2).
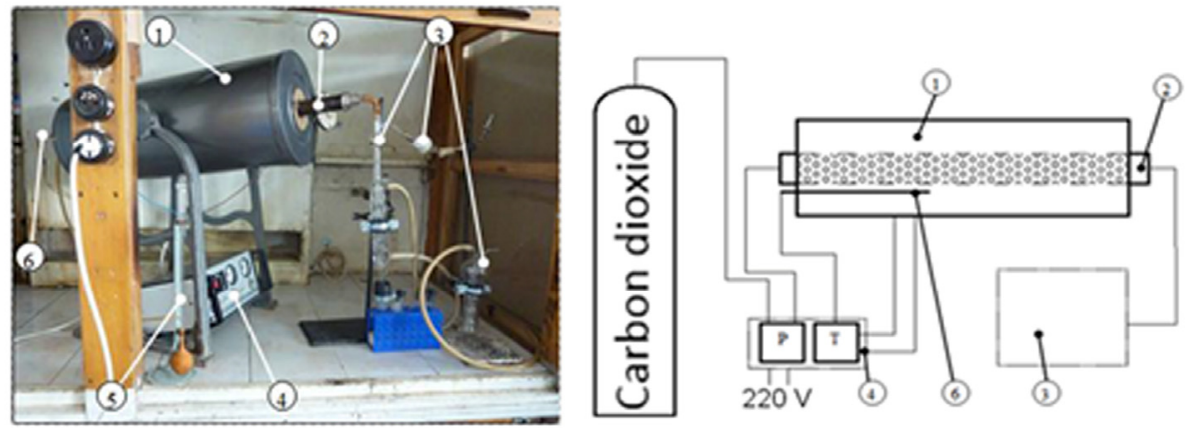

Fig. 2. Experimental pyrolysis plant for oil-sorbent preparing: 1 - tube furnace; 2 - pyrolyser reactor; 3 - cooling and gas cleaning system; 4 - control unit; 5 - foam flowmeter; 6 - temperature sensor.

The heating rate of the furnace is $15-25{ }^{\circ} \mathrm{C}$, the maximum temperature is $1000{ }^{\circ} \mathrm{C}$. The reactor of the pyrolysis furnace is a cylindrical steel vessel (internal diameter $27 \mathrm{~mm}$, length $820 \mathrm{~mm}$ ) with a useful volume of $4.7 \times 10^{-4} \mathrm{~m}^{3}$, equipped with fittings at both ends: for introducing inert gas and for removing the vapor-gas mixture.

Loading of the reactor-pyrolyzer took place in $2 / 3$ of the volume, and then it was placed in a tube furnace for heating for 30 minutes. After the end of pyrolysis, the plant was turned off; an inert carbon dioxide-containing agent was supplied to the reactor for cooling.

The magnetic sorbent was tested as follows: oil was applied to the water surface in such a way that oil occupies the entire surface. A granule of a known mass was placed on the imitated spill and the sorption process was observed. After 24 hours, the oil -sorbent pellets were weighed, dried for 3 hours at a temperature of up to $35^{\circ} \mathrm{C}$ (an increase in temperature 
is not permissible, since the gasoline fraction escapes from oil). The moisture capacity and oil capacity of the magnetic oil-sorbent were determined in experiment on the "oil-water" phase section. In a similar way, a separate determination of the moisture capacity and oil capacity in conditions of one phase, or "water", or "oil," is determined.

\section{Results and discussion}

The main properties of used raw materials are shown in Table 1.

Table 1. Properties of used raw materials

\begin{tabular}{|l|c|c|c|}
\hline \multicolumn{1}{|c|}{ Component } & Humidity, $\%$ & Ash content, $\%$ & Density, $\mathbf{~} \mathbf{\%} / \mathbf{m}^{\mathbf{3}}$ \\
\hline Biomass & 80.6 & 21.1 & 1,048 \\
\hline $\begin{array}{l}\text { Coal dust } \\
\text { (cyclone) }\end{array}$ & 0.6 & 24.5 & 760 \\
\hline Magnetite & 3.0 & 100.0 & 2,800 \\
\hline
\end{tabular}

The moisture content of the biomass in anaerobic digestion was selected in such a way that combustible biogas with a methane content of more than $65 \%$ was released.

Thus, for molded granules producing we use a binder with the following characteristics: humidity $-89.2 \%$ wt., ash content $-24.2 \%$ wt., density $-1021 \mathrm{~kg} / \mathrm{m}^{3}$.

According to laboratory tests, to obtain the optimum sorbent composition, it is necessary to use $21 \%$ wt. binder in the mixture for pelletizing. With a given amount of binder, the maximum yield of the desired product after pyrolysis corresponds to the processing of molded granules of diameter 2-10 mm. This is the minimum amount of binder necessary for the granulation process. With a lack of a binder, a cellular structure is formed. It should be noted that with an overabundance of the binder, the mixture adheres to the internal surface of the apparatus, which reduces the efficiency of the rolling process.

There are advantages and disadvantages in various ways of magnetite introduced. The method of powdering the finished shaped pellets with magnetite is the simplest in the implementation and allows the components to be evenly distributed over the granule. However, in this case, the "magnetite shell" peeled off by mechanical action also creates an obstacle to the penetration of oil into the granule sorbing carbonaceous material. Therefore, this method of introducing magnetite is not recommended.

The option of introducing magnetite into the granulation mixture causes the need for more thorough mixing to distribute the components evenly. Advantages of the method used: magnetite particles do not interfere with the sorption of oil and oil products, remain in the oil sorbent granule throughout the entire period of use.

Theoretically, "magnetite core" is the most effective, because the most expensive component is initially represented in the core of granules, and, consequently, its losses are minimized. Secondly, such a nucleus is easy to reuse (the spent sorbent is burned using heat, and the magnetite cores are separated from the ash by means of a magnet). However, in order to realize this method, it is necessary to search for a binder that does not do the magnetite heavier, is stable at temperatures up to $600{ }^{\circ} \mathrm{C}$ without air access (during pyrolysis), and does not burn at temperatures up to $1,000{ }^{\circ} \mathrm{C}$. Since such a binder is not found, it is considered optimal to use magnetite distributed over the volume of granules.

In result of pyrolysis, the granules do not lose their original form, although the strength of the granules decreases by a factor of 2 (compressive strength of the initial granules is 1,500 $\mathrm{g}$, the finished sorbent $-680 \mathrm{~g}$.). However, it is worth noting that the introduction of magnetite has virtually no effect on the strength of the sorbent (when its content in the mixture is up to $15 \% \mathrm{wt}$.).

Within the framework of the laboratory study, an experiment of the dependence attractive 
force of the obtained oil-sorbents with different magnetite content from the distance to the magnet was performed (Figure 3).

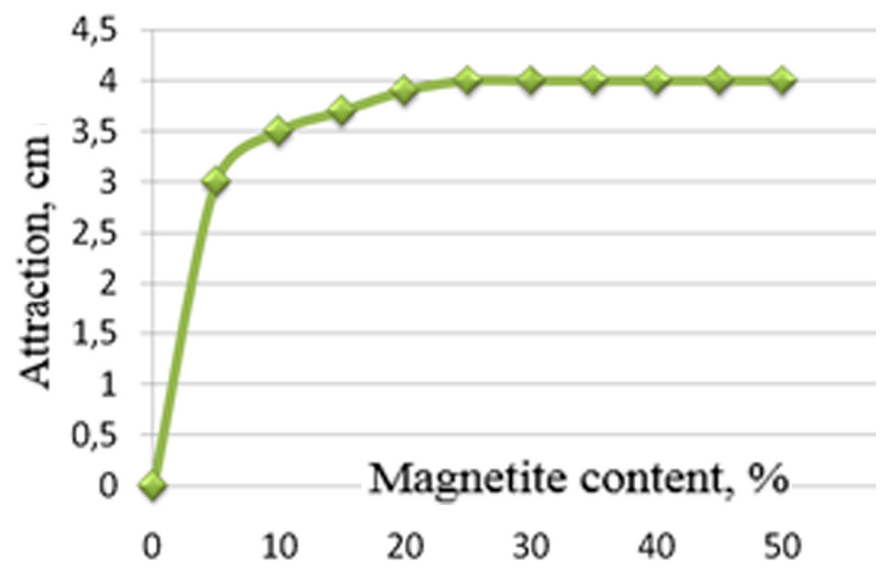

Fig. 3. The graph of the force of attraction (the distance from the magnet to the sorbent) dependence on the content of magnetite in the oil-sorbent.

As seen with 5-10\% wt. containing of magnetite, attraction to the magnet is observed at a distance of $37 \mathrm{~mm}$, after additional introduction of magnetite this value practically does not change. In practice, to increase the distance from the magnet to the oil-sorbent, it is necessary to increase the magnetic field strength or choosing another magnet material.

Below is a comparison of the characteristics of the oil-sorbent with the introduction of magnetite and without it (Table 2). From practical data it can be seen that the magnetic sorbent is not inferior in performance to the usual one. A significant difference can be seen in the parameter ash content, since magnetite enters the inorganic part of the sorbent and during combustion passes into ash.

Table 2. Comparison of magnetic and conventional sorbent characteristics

\begin{tabular}{|l|c|c|}
\hline \multicolumn{1}{|c|}{ Parameter } & $\begin{array}{c}\text { Conventional } \\
\text { sorbent }\end{array}$ & $\begin{array}{c}\text { Magnetic } \\
\text { sorbent }\end{array}$ \\
\hline Humidity, \% & 2.0 & 2.8 \\
\hline Ash, \% & 22.4 & 30.6 \\
\hline Oil capacity, g/g & 3.5 & 3.0 \\
\hline Moisture capacity, g/g & 2,1 & 1.6 \\
\hline The yield of volatile substances , \% & 35.2 & 33.3 \\
\hline Density, $\mathrm{K} \Gamma \mathrm{M}^{3}$ & 195.0 & 345.0 \\
\hline Compressive strength, g/granule & 520.0 & 550.0 \\
\hline Buoyancy, days & 20 & 20 \\
\hline Bulk density, $\mathrm{Kg} / \mathrm{m}^{3}$ & 151.0 & 165.0 \\
\hline Adsorption capacity for iodine, g/g & 4.3 & 4.2 \\
\hline Additional characteristics & - & Magnetically controlled \\
\hline
\end{tabular}

The possibility of extracting magnetite from the spent sorbent after combustion is very important: the resulting ash is placed in water and thoroughly mixed to "wash" magnetite from the ash; the pure magnetite is subsequently collected by an electromagnet and sent for reuse.

In case of using of magnetic sorbent in large reservoirs, the use of existing single-hull magnetic vessels is recommended, the basic scheme of which is shown in Figure 4 [12]. 


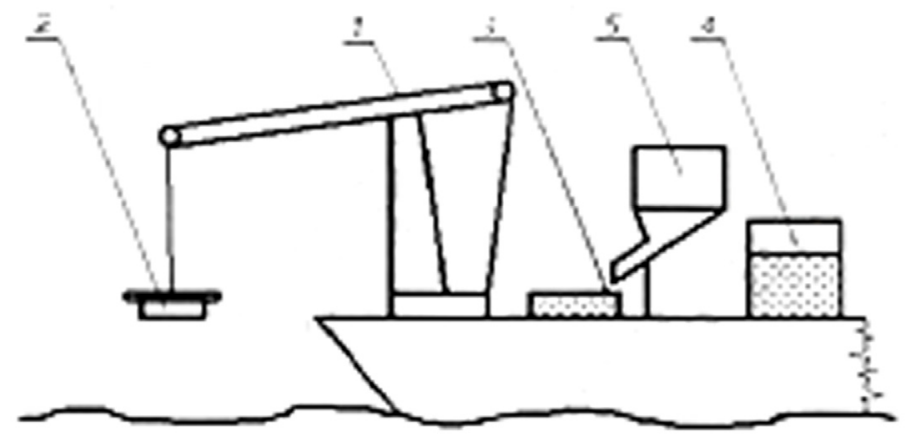

Fig. 4. Scheme single-hull vessel: 1 - lifting device; 2 - an electromagnetic washer; 3 - initial adsorbent; 4 - assembled tank; 5 - bunker with initial sorbents .

The device for collecting the saturated oil-sorbent works as follows: the electromagnetic washer attracts a predetermined amount of the initial sorbent from the container 3 and places it on a layer of spilled hydrocarbons for 5-7 minutes. After the sorbent is saturated with a magnetic washer, it is removed from the oil product layer and transferred to a collecting container 4 above which the electric power supply of the washer is disconnected and the saturated sorbent falls into the collecting container under its own weight. Then the cycle repeats.

\section{Conclusion}

The use of coal mining waste, in particular coal dust, in the production of sorbents is possible, while its content can vary in a wide range. However, the optimal composition is: $75 \% \mathrm{wt}$. coal mining and coal processing waste, $20 \%$ wt. binder, and 5\% magnetite.

\section{References}

1. State report "On the state and on the protection of the environment of the Russian Federation in 2015" 639 (M.: Ministry of Natural Resources of Russia; NNA-Nature. - 2016)

2. Andrey Ushakov, Elena Ushakova, Gennadiy Ushakov, Taishan Academic Forum Project on Mine Disaster Prevention and Control, 445 (2014)

3. Andrey Ushakov, Alternative energy and ecology, 7, 106 (2011)

4. Elena Briukhanova, Gennadiy Ushakov, Andrey Ushakov, Alternative energy and ecology, 5, 71 (2010)

5. Andrew Nikiforuk, Coastal Science and Societies, A Brief Overview of the Oil Spill Problem in Russia (2016)

6. Oil tanker spill statistics, Annual report of International Tanker Owners Pollution Federation Limited (ITOPF) (2016)

7. Mervin Fingas, Oil Spill Science and Technology, 1078 (Gulf Professional Publishing, 2016)

8. Stephanie E. Chang, Jeremy Stone, Kyle Demes, Marina Piscitelli, Ecology and Society, 19:2, (2014)

9. E.R. Gundlach, Journal of Coastal Research, 39, 39 
10. Elena Briukhanova, Processes for oil-sorbent obtaining by pyrolysis of pellets based on wood waste and organic binder in layered apparatus, 152 (Tomsk, 2013)

11. Flores Arias, Development of a sorbent with magnetic properties based on iron oxides and metallurgical waste for liquidation of oil spills, 137 (Belgorod, 2012) 
